\title{
RAPID HIGH-PERFORMANCE THIN LAYER CHROMATOGRAPHIC QUANTITATIVE ESTIMATION OF CAFFEINE IN VARIOUS FOODS AND BEVERAGES
}

\author{
Rohit Raj ${ }^{1}$, K. S. Chandrashekar ${ }^{1}$, Rounak Biswas ${ }^{1}$, Aravind Pai ${ }^{2}$ \\ and Vasudev Pai ${ }^{1}$ ® \\ ${ }^{1}$ Department of Pharmacognosy, Manipal College of Pharmaceutical Sciences, Manipal \\ Academy of Higher Education, Manipal, Karnataka, India-576104 \\ ${ }^{2}$ Department of Pharmaceutical Chemistry, Manipal College of Pharmaceutical Sciences, \\ Manipal Academy of Higher Education, Manipal, Karnataka, India-576104 \\ ${ }^{\square}$ Corresponding Author: pai.vasudev@manipal.edu
}

\begin{abstract}
Caffeine is an alkaloid and well-known CNS stimulant usually added in most foods and beverages. Caffeine had drawn more attention in many nutritional products due to its stimulant effect on CNS, so that the product to which caffeine is added becomes more popular in the market. The present work was carried out to extract and estimate the amount of caffeine in the various food and beverages by chromatography technique using HighPerformance Thin Layer Chromatography. Procured marketed products like confectioneries, foods and beverages are subjected for extraction and estimated the caffeine content by HPTLC method by using mobile phase Ethyl acetate: Methanol (9:1) and quantified. Monster and Tzinga both energy drinks showed the content within the label claim (23.96 and $25.43 \%$ respectively). However other products though they did not have a label claim, showed significant caffeine content, in that dark chocolate showed the highest $11.40 \%$, whereas diet coke did not show any caffeine content. The consumable food products like biscuits, energy drinks, and other beverages with added caffeine should have quantitative labeling on the wrapper to help the consumer to know about the caffeine. But label claim of caffeine is missing on some products, HPTLC method helps to estimate the caffeine content from the marketed products.
\end{abstract}

Keywords: Caffeine, CNS Stimulant, Food and Beverages and HPTLC.

RASĀYAN J. Chem., Vol. 14, No.1, 2021

\section{INTRODUCTION}

Food is a very important and essential part of our life, as a human being is dependent on food for energy. Hippocratic thought, "Let food be the medicine and medicine be the food". Food is usually sourced from plants and animals which contains basic essential nutritional supplements such as carbohydrates, fats, proteins, vitamins and minerals. ${ }^{1}$ At the same time beverages or drinks have also been occupied an important space in human nutritional consumption along with food especially for quick energy and CNS stimulation. ${ }^{2}$ Hot and cold beverages like tea, coffee, hot chocolate, energy drinks, soft drinks, cold coffee etc. have become an integral part of our routine diet. ${ }^{3}$ Number of foods and beverages which are used in our day to day life and which gives fast energy are known to contain caffeine which is a CNS stimulant and a habit-forming constituent. ${ }^{4}$

Caffeine is a psychoactive phytoconstituent obtained from different plants like Coffee, Tea etc. "Caffeine" originated from the German word "kaffee" and the French word "cafe" meaning of both is coffee. ${ }^{5}$ Now day's caffeine has become an integral part of the day to day life. Caffeine is known as the most commonly utilized psychoactive substance in the world. ${ }^{6}$ Common sources of caffeine include coffee, tea, caffeinated soft drinks, energy drinks and to a lesser extent chocolates derived from cocoa beans. ${ }^{7}$ Caffeine tablets manufactured by various companies are emerging as a new trend and as an alternative to energy drinks.

Caffeine is a white crystalline xanthine alkaloid and is bitter and has a stimulant action on CNS. Caffeine is a common name for 1,3,7-trimethyl xanthine. The source of caffeine is obtained from the coffee plant, kola nut, guarana berries, guayusa and yaupon holly. ${ }^{8}$ The xanthine molecule of caffeine has two fused rings of pyrimidinedione and imidazole. The pyrimidinedione contains two amide 
groups that exist in a zwitterion resonance form where the nitrogen atoms are double bonded to their adjacent amide carbons atoms. ${ }^{9}$

\section{Pharmacology}

Adenosine neurotransmitter is inhibitory action that suppresses CNS activity. Caffeine is structurally similar to adenosine and therefore it binds to adenosine receptors resulting in blockage of adenosine receptors. ${ }^{10}$ Caffeine like other xanthines also acts as a competitive, nonselective phophodiesterase inhibitor that raises intracellular cAMP, activates protein kinase A, inhibits $\mathrm{TNF} \alpha$, leukotriene and reduces inflammation and innate immunity. ${ }^{11}$

\section{Health Benefits}

Caffeine is a CNS stimulant and also stimulates the metabolic process. It is widely used both medicinally and for leisure to diminish physical exhaustion or to overcome alertness during drowsiness. It produces expanded alertness, more clear progression of thought, increased focus, and better coordination. The caffeine content required to produce desired effects varies from individual to individual, depending upon body size and level of resilience. Caffeine effects begin within a short time on administration and the effect usually remains around five hours. Caffeine has various impacts on sleep, but again it does not show the same effect on all individuals. It improves execution during lack of sleep but later leads to subsequent sleeplessness or insomnia. ${ }^{12}$

\section{Adverse Effects}

Too much caffeine may lead to restlessness, anxiousness and irritability. (US National Institute of health). Caffeine overdose (more than $400-500 \mathrm{mg}$ at a time can result in overstimulation of CNS called caffeine intoxication. Consumption of caffeine in higher doses may lead to caffeine-induced sleep disorders. (American Psychiatric association 1994)

Addiction: People who take at least $100 \mathrm{mg}$ of caffeine in a day (about 1 cup of coffee per day) can get physical dependence that would trigger withdrawal indications which include headache, muscle stiffness and pain, nausea and vomiting, lethargy, depressed mood and irritation.

Therefore, it becomes very important to know the caffeine content of every food product, beverages and drinks we consume so that we can limit our daily caffeine intake and avoid the adverse effects of caffeine.

Several estimations have been carried out on caffeine in various foods and beverages. Therefore, it was thought worthwhile to estimate the caffeine content.

Caffeine is widely used since time immortal in the form of tea and coffee. It is used as a natural central nervous system stimulant. Chemically it is a xanthine derivative that occurs naturally and is extracted from leaves (Thea sinensis), seeds (Coffee Arabica and other species of Coffee) and cocoa. It is the most widely consumed psychoactive substance got respect by almost all cultures due to its ability to keep alert and is legal and unregulated unlike other psychoactive drug. According to survey done in North America it was found that nearly $90 \%$ of adults consume coffee every day in various forms of a drink.

Caffeine and pregnancy: Pregnant women who drink large amounts of caffeine-containing drinks should be aware of its side effect because during pregnancy half-life of caffeine is more than nonpregnant individuals according to a study half-life of caffeine was done in saliva sample of the individual which shows mean. ${ }^{13}$

\section{EXPERIMENTAL}

Caffeine estimation was done in different food and beverages available in the market and the aim for estimating the caffeine content is to compare it with label claim. Marketed samples were taken for caffeine estimation: Bourbon biscuit, Dark chocolate, Cadbury Oreo biscuit, Diet coke, Monster, Tzinga, Tetley green tea.

Extraction of Caffeine from Monster, Tzinga and Diet coke: $10 \mathrm{ml}$ of the marketed sample of Monster was pipetted out into a separating funnel and extracted with $15 \mathrm{ml}$ of chloroform. The chloroform layer was separated and again extracted twice with $10 \mathrm{ml}$ chloroform. The extract was taken in a china dish and evaporated in a water bath.

\section{Extraction of Caffeine from Tetley Green Tea}

Mix $25 \mathrm{~g}$ of sample and $2.5 \mathrm{~g}$ of anhydrous sodium carbonate with $150 \mathrm{ml}$ of water in a $250 \mathrm{ml}$ beaker and boil gently for 10 minutes. Strain the hot solution through a muslin cloth. Wash the marc with 
further $20 \mathrm{ml}$ of boiling water. Cool the extract to room temperature and transfer it into a separating funnel. Add $15 \mathrm{ml}$ of dichloromethane to a separating funnel and shake to avoid the formation of an emulsion. Allow the content to separate. The dichloromethane layer is collected and the aqueous content is again extracted with another quantity $10 \mathrm{ml}$ and repeat the procedure. The dichloromethane extract is collected and evaporated to dryness to collect caffeine.

Extraction of Caffeine from Bourbon, Dark chocolate, and Cadbury Oreo: $12 \mathrm{~g}$ of Cadbury Oreo was taken and defatted 2 times with Petroleum ether and filtered. The filtrate was extracted with Methanol and partitioned initially with $15 \mathrm{ml}$ Chloroform and then $10 \mathrm{ml}$ Chloroform twice. The Chloroform layer was taken in a china dish and evaporated in a water bath.

\section{Instruments and Materials Used}

Digital weighing balance (Denver, U.S.A), Sonicator (Equitron, Labnet Scientific Services, India), CAMAG HPTLC System (CAMAG, Switzerland), LINOMAT-V Automatic Sample Applicator, CAMAG TLC Scanner-3, CAMAG Reprostar-3 Documentation System, Hamilton Syringe (100 $\mu 1$ capacity, U.S.A).

Chemicals: All chemicals used were of analytical grade like Petroleum Ether, Chloroform, Methanol, Ethyl acetate, dichloromethane.

\section{HPTLC Parameter}

Standard marker:

Stationary Phase:

Mobile Phase:

Developing chamber:

Saturation time:

Migration Distance:

Concentration of Standard:

Concentration of Sample:

Sample volume/track:

Band length:

Detection wavelength:
Caffeine (99\% pure) (Sigma Aldrich)

Pre-coated HPTLC Silica Gel GF $254(10 \mathrm{~cm} \times 10 \mathrm{~cm})$

Ethyl acetate: Methanol (9: 1)

Camag twin trough glass chamber $(20 \times 10 \mathrm{~cm})$

30 Minutes

$80 \mathrm{~mm}$

$5 \mu \mathrm{g} / \mathrm{ml}$

$5 \mathrm{mg} / \mathrm{ml}$

$4 \mu 1$

$6 \mathrm{~mm}$

$273 \mathrm{~nm}$

Percentage of caffeine content was determined by using the following formula

$$
\% \text { Content of caffeine }=\frac{\text { AUC of Sample X Con.c of standard X \% purity of Standard }}{\text { AUC of standard X Con.c of sample }}
$$

Results of caffeine content in different marketed products are given in Table 1. Figure 1 shows 3D Chromatogram of all the products and caffeine.

\section{RESULTS AND DISCUSSION}

The HPTLC estimation of caffeine in various food and beverages showed the presence of caffeine in all except in diet coke did not show any caffeine content. Monster and Tzinga both energy drinks showed the content within the label claim (23.96 and 25.43\% respectively). However other products though they did not have a label claim, showed significant caffeine content, in that dark chocolate showed the highest $11.40 \%$. The present study aimed to estimate caffeine in various food products and beverages. In our study, we obtained some of the food products and beverage is known to contain caffeine from the market. HPTLC is an accurate and most important analytical tool to estimate various phytoconstituents and hence used for this study. From our study, it was observed that food products and beverages contain caffeine. Tzinga and monster showed caffeine content within in the label claim. While few other products like dark chocolate, Oreo biscuit, Burbon cream biscuit, green tea although they did not claim a significant percentage of caffeine were found, while diet coke did not show any caffeine content. The presence of caffeine shows the addition of the same direction or maybe due to chocolate which is made from cocoa. Cocoa is known to contain caffeine.

Table-1: Estimation of Caffeine Content

\begin{tabular}{c|c|c|c|c}
\hline S.No. & Fraction & AUC & \% Content of Caffeine & Label Claim (\% Content) \\
\hline 1 & Std. Caffeine & 13257.5 & -- & -- \\
\hline 2 & Bourbon & 976.8 & 7.30 & -- \\
\hline
\end{tabular}


RASĀYAN J. Chem.

Vol. 14 | No. 1 |221-226| January - March | 2021

\begin{tabular}{c|c|c|c|c}
\hline 3 & Diet coke & -- & Not detected & -- \\
\hline 4 & Dark chocolate & 1524.5 & 11.40 & -- \\
\hline 5 & Green tea & 12675.15 & 9.465 & -- \\
\hline 6 & Monster & 3209.1 & 23.96 & 32 \\
\hline 7 & Tzinga & 3405.5 & 25.43 & 30 \\
\hline 8 & Cadbury Oreo & 410.2 & 3.06 & -- \\
\hline
\end{tabular}

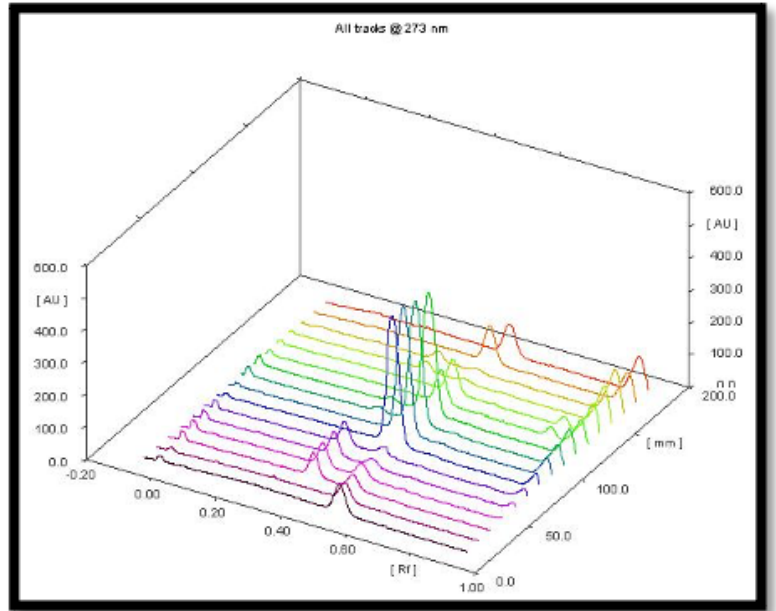

Fig.-1: 3D Chromatogram of Caffeine in all the Products and Standard Caffeine

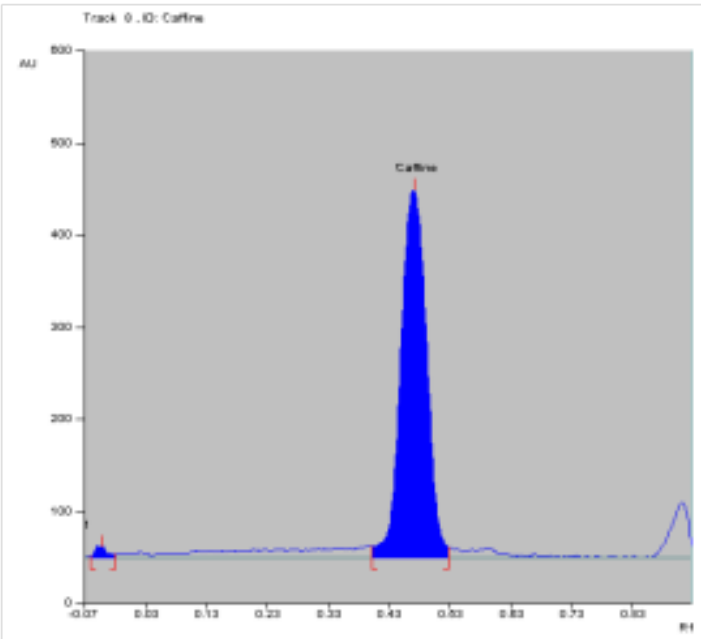

Fig.-2: Peak of Standard Caffeine

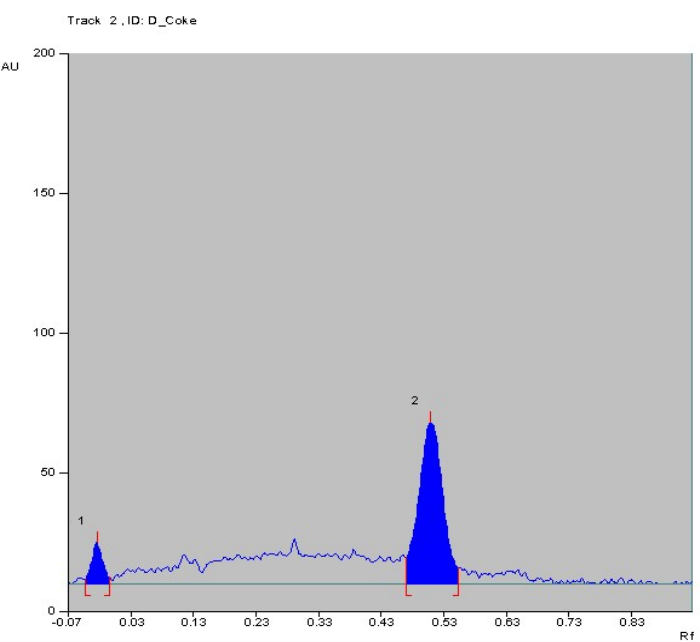

Fig.-4: Caffeine in Coke

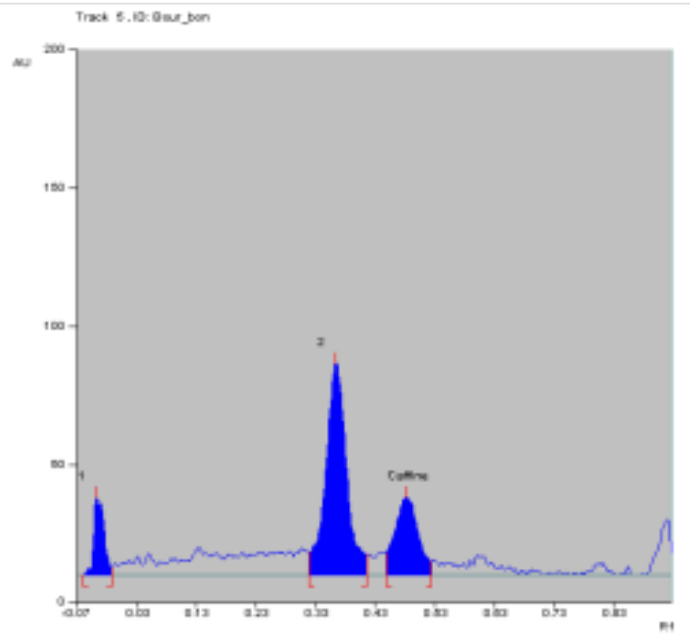

Fig.-3: Caffeine in Bourbon

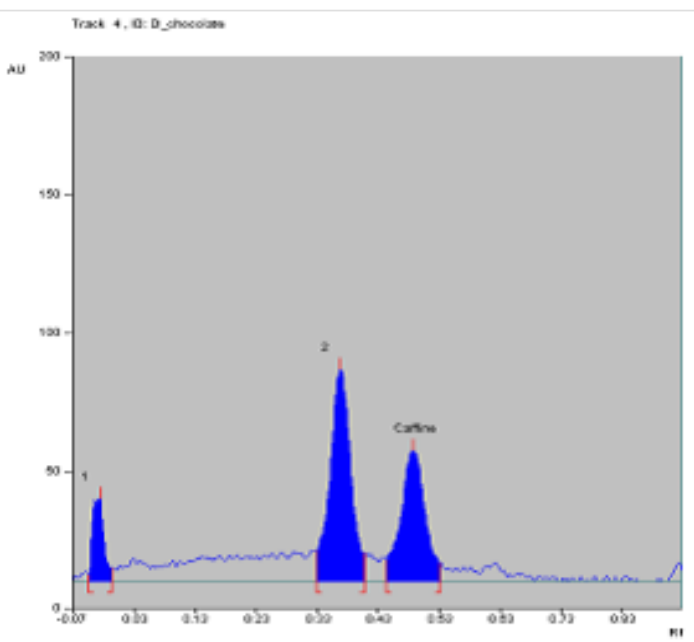

Fig.-5: Caffeine in Dark Chocolate 


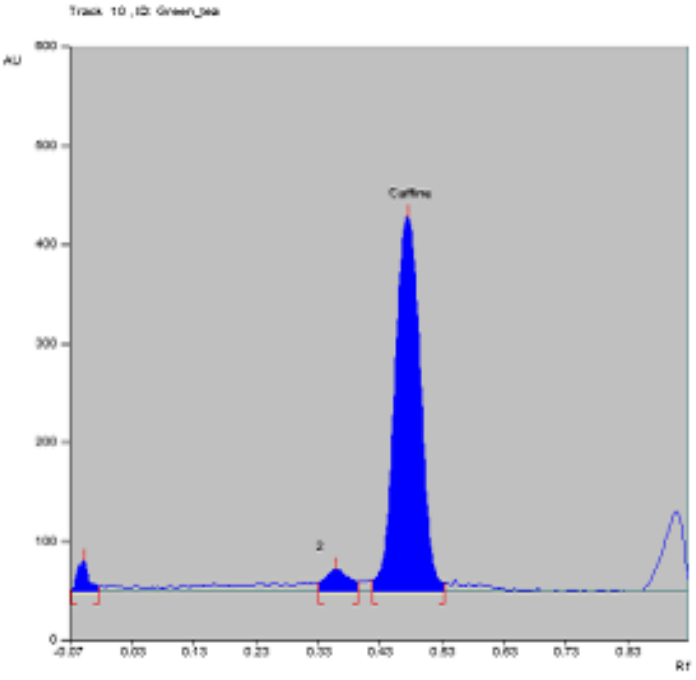

Fig.-6: Caffeine in Green Tea

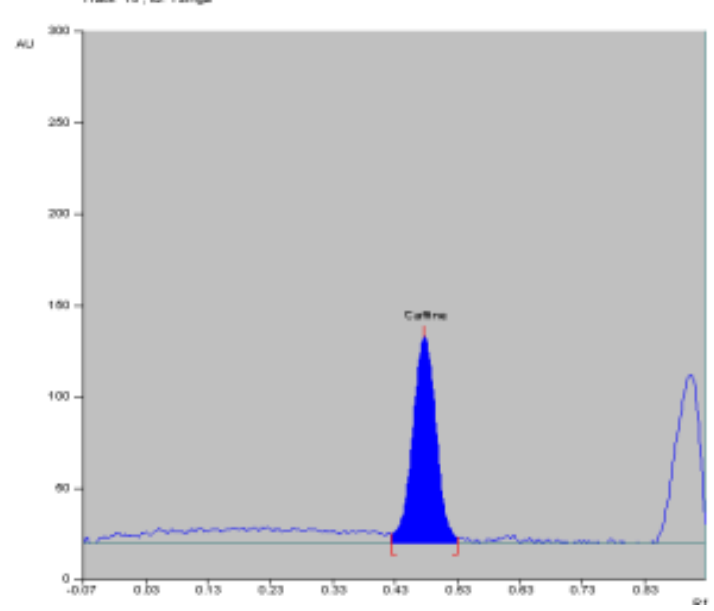

Fig.-8: Caffeine in Tzinga

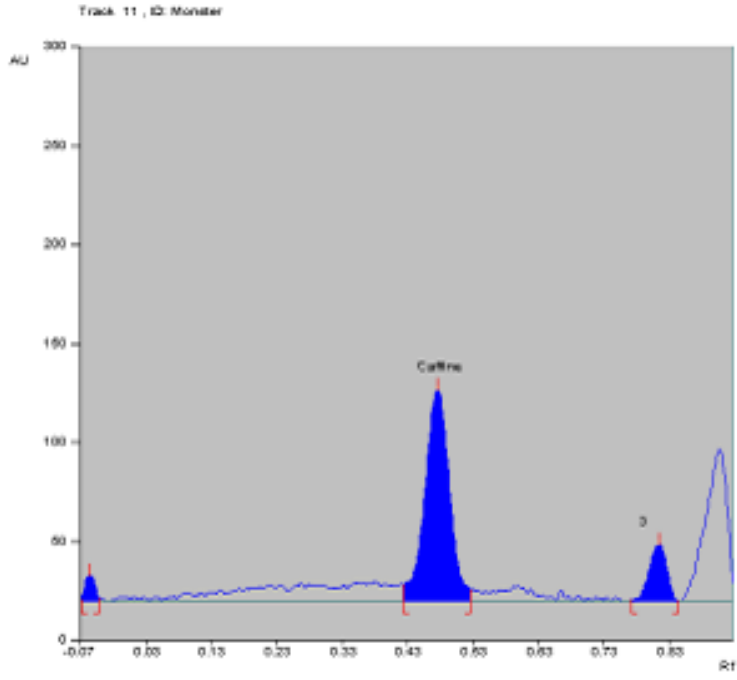

Fig.-7: Caffeine in Monster

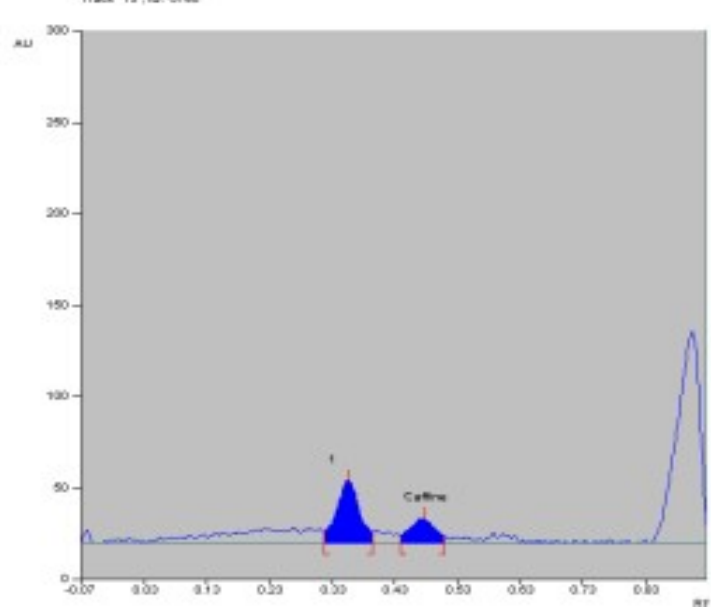

Fig.-9: Caffeine in Oreo Spectra comparison

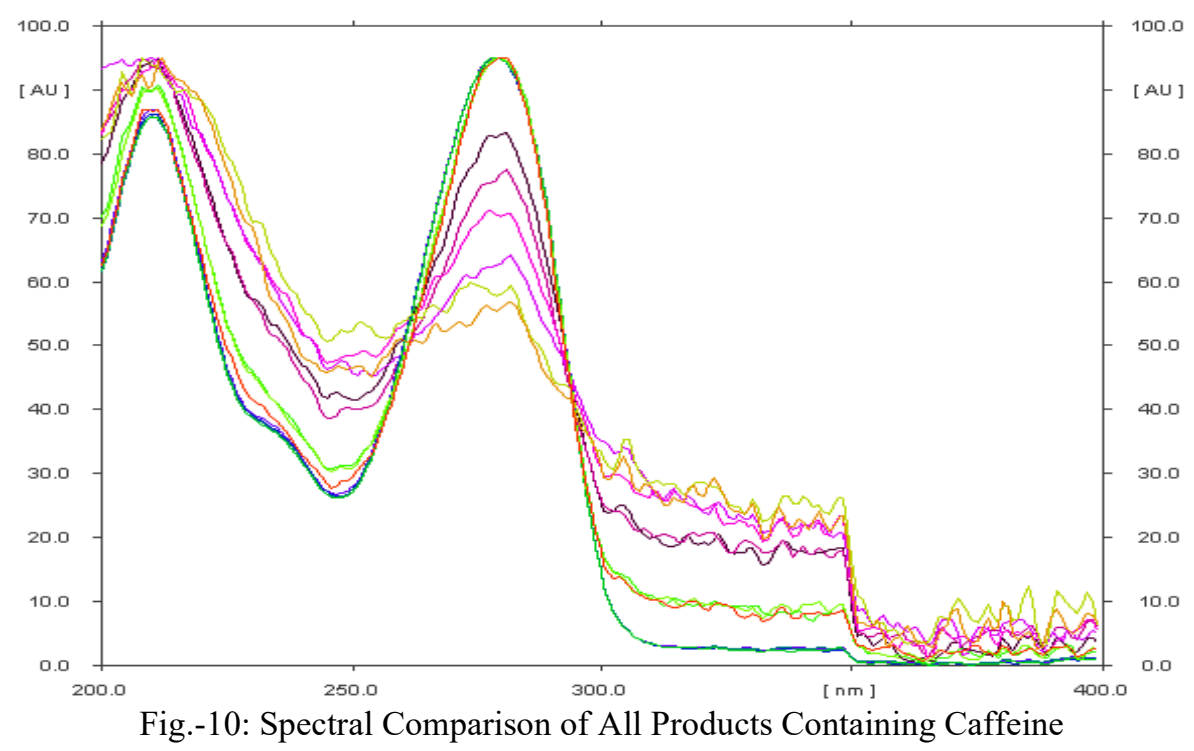

\section{CONCLUSION}

The consumable food products like biscuits, energy drinks, and other beverages with added caffeine should have quantitative labeling on the wrapper to help the consumer to know about the caffeine. This prevents serious harm, protects children and adolescents and also helps safe consumer use. Even though the content of caffeine is at the limit it is right of the consumer to know how much caffeine they are consuming. 


\section{ACKNOWLEDGMENT}

The authors are thankful to the Department of Pharmacognosy, Manipal College of Pharmaceutical Sciences and Manipal Academy of Higher Education for providing laboratory facilities and other infrastructure to carry out the work.

\section{REFERENCES}

1. R.F.Witkamp, K.Van Norren, European Journal of Pharmacology, 836, 102(2018), DOI: $10.1016 /$ j.ejphar.2018.06.026

2. D. C. Mitchell, C. A. Knight, J. Hockenberry, R. Teplansky, T. J. Hartman, Food and Chemical Toxicology, 63,136(2014), DOI:10.1016/j.fct.2013.10.042

3. Z.M.Chen, Z.Lin, Journal of Zhejiang University-Science B, 16(2), 87(2015), DOI: 10.1631 jzus.B1500001

4. S. Cappelletti, P. Daria, G. Sani, Current Neuropharmacology,13(1),71(2015), DOI: $10.2174 / 1570159$ X13666141210215655

5. M.A.Heckman, J.Weil, E.G.De Mejia, Journal of Food Science, 75(3), R77-87(2010), DOI: $10.1111 / \mathrm{j} .1750-3841.2010 .01561 . \mathrm{x}$

6. B.A.Weinberg, P.D.Weinberg, B.K.Bealer, The World of Caffeine: The Science and Culture of the World's Most Popular Drug, Psychology Press, 2001.

7. A. Drewnowski, C.D.Rehm, Nutrients, 8(3), 154(2016), DOI:10.3390/nu8030154

8. B.B.Kasimala, M. Babu Kasimala, International Journal of Pharmaceutical \& Biological Archives, 2(6), 1757(2011).

9. N. Keskineva, "Chemistry of Caffeine" (PDF), Chemistry Department, East Stroudsburg University. Retrieved 10 January 2019

10. H. Meyers, Suave Molecules of Mocha-Coffee, Chemistry, and Civilization, New Partisan, 3(07), 2005

11. M. Peters-Golden, C. Canetti, P. Mancuso, M.J.Coffey, The Journal of Immunology, 174(2), 589(2005), DOI:10.4049/jimmunol.174.2.589

12. J. Snel, M.M.Lorist, Progress in Brain Research,190, 105(2011), DOI:10.1016/B978-0-44453817-8.00006-2

13. R. Knutti, H. Rothweiler, C. Schlatter, The Effect of Pregnancy on the Pharmacokinetics of Caffeine, In New Toxicology for Old 1982 (pp. 187-192), Springer, Berlin, Heidelberg.

[RJC-5991/2020] 